\title{
Perfil da mulher portadora de HIV/AIDS e sua adesão ao tratamento
}

\author{
Profile of women with HIV/AIDS and their adherence to treatment \\ Perfil de las mujeres con VIH/SIDA y su adherencia al tratamiento
}

Shirlley Sayonara Bezerra de Melo Torres ${ }^{1 *}$, José Ednilson Edmilson de Melo², Angélica de Godoy Torres Lima ${ }^{3}$, Jaciele Cristina da Silva Belone ${ }^{3}$, Marilene Cordeiro do Nascimento ${ }^{3}$, Rebeca Larissa Silva $^{4}$, Thamyris Vieira de Barros ${ }^{4}$, Eliane Braz da Silva Arruda ${ }^{5}$, Thaciana Izidoro Patrício ${ }^{6}$, Cristina Rosane Jordão Braga Vilaça ${ }^{7}$.

\section{RESUMO}

Objetivo: Avaliar o perfil da mulher portadora de HIV/AIDS e sua adesão ao tratamento em um serviço de referência de um município de Pernambuco. Métodos: Trata-se de estudo transversal descritivo, quantitativo, entre janeiro de 2014 a dezembro 2017. Inicialmente havia uma lista com 2.100 pacientes infectados pelo HIV/aids. Foram selecionadas as mulheres, objeto do estudo, totalizando 211 portadoras desta patologia. Sendo que, 12 pacientes não foram incluídas no estudo por serem menores de 18 anos, restando 199 o número de participantes desta pesquisa. Destas, 14 não aderiram ao tratamento, 9 desistiram do tratamento e 4 foram diagnosticadas mais não quiseram aderir ao tratamento, segundo registro em prontuário. $O$ estudo foi aprovado por Comitê de Ética em Pesquisa com número de parecer 1.868.426. Resultados: A idade média das mulheres do estudo ficou entre 30 e 39 anos, metade estudaram até o ensino fundamental $(59,8 \%)$, se declaram pardas $(64,3 \%)$ e eram solteiras $(56,8 \%)$. A principal forma de aquisição da infecção foi por relação sexual $(69,8 \%)$. Considerações finais: A identificação do perfil e especificidades dessa população de estudo, permite que se criem planos de ação e políticas direcionadas, objetivando obter melhoria na adesão ao tratamento, podendo gerar redução de gastos e benefícios desta clientela.

Palavras-chave: Mulher, HIV/AIDS, Adesão.

\begin{abstract}
Objective: To evaluate the profile of women with HIV/AIDS and their adherence to treatment in a reference service in a municipality of Pernambuco. Methods: This is a descriptive, quantitative cross-sectional study between January 2014 and December 2017. Initially there was a list of 2,100 HIV/AIDS-infected patients. Women were selected, the object of the study, totaling 211 carriers of this pathology. Of course, 12 patients were not included in the study because they were under 18 years of age, with 199 remaining the number of participants in this study. Of these, 14 did not adhere to treatment, 9 gave up treatment and 4 were diagnosed but did not want to adhere to treatment, according to medical records. The study was approved by the Research Ethics Committee with an opinion number of 1,868,426. Results: The mean age of the women in the study was between 30 and 39 years, half studied until elementary school (59.8\%), declare themselves brown (64.3\%) and were single (56.8\%). The main form of acquisition of the infection was by sexual intercourse $(69.8 \%)$. Final considerations: The identification of the profile and specificities of this study population allows the creation of action plans and targeted policies, aiming to obtain improvement in treatment adherence, which can generate a reduction in the costs and benefits of this clientele.
\end{abstract}

Keywords: Woman, HIV/AIDS, Adherence

\footnotetext{
${ }^{1}$ Centro de Formação, Pós-graduação e Pesquisa em saúde (CEFAPP), Caruaru - PE.

*E-mail: shirlley_sayonara@hotmail.com

${ }^{2}$ Centro Universitário Internacional Uninter (UNINTER), Caruaru - PE.

${ }^{3}$ Instituto Federal de Educação, Ciência e Tecnologia de Pernambuco (IFPE), Belo Jardim - PE.

${ }^{4}$ Centro Universitário Vale do Ipojuca (UNIFAVIP/Devry), Caruaru - PE.

5 Universidade Federal de Pernambuco (UFPE), Vitória de Santo Antão - PE.

'Universidade Federal do Maranhão (UFMA), São Luiz - MA.

${ }^{7}$ Universidade de Pernambuco (UPE), Recife - PE.
} 


\section{RESUMEN}

Objetivo: Evaluar el perfil de las mujeres con VIH/SIDA y su adhesión al tratamiento en un servicio de referencia en un municipio de Pernambuco. Métodos: Se trata de un estudio transversal descriptivo y cuantitativo entre enero de 2014 y diciembre de 2017. Inicialmente había una lista de 2.100 pacientes infectados por el VIH/SIDA. Las mujeres fueron seleccionadas, objeto del estudio, con un total de 211 portadores de esta patología. Por supuesto, 12 pacientes no fueron incluidos en el estudio porque eran menores de 18 años, quedando 199 el número de participantes en este estudio. De ellos,14 no se adhirieron al tratamiento, 9 renunciaron al tratamiento y 4 fueron diagnosticados, pero no quisieron adherirse al tratamiento, según los registros médicos. El estudio fue aprobado por el Comité de ética de la investigación con un número de opinión de 1.868.426. Resultados: La edad media de las mujeres en el estudio fue de entre 30 y 39 años, la mitad estudiada hasta la escuela primaria $(59,8 \%)$, se declaran marrones $(64,3 \%)$ y eran individuales $(56,8 \%)$. La principal forma de adquisición de la infección fue por relación sexual $(69,8 \%)$. Consideraciones finales: La identificación del perfil y las especificidades de la población de este estudio permite la creación de planes de acción y políticas específicas, con el objetivo de obtener una mejora en la adherencia al tratamiento, lo que puede generar una reducción en los costes y beneficios de esta clientela.

Palabras clave: Mujer, VIH/SIDA, Adherencia.

\section{INTRODUÇÃO}

A infecção pelo vírus da imunodeficiência humana (HIV) e sua manifestação clínica em fase avançada, a síndrome da imunodeficiência adquirida (AIDS), ainda representam um problema de saúde pública de grande relevância na atualidade, em função do seu caráter pandêmico e de sua transcendência. As pessoas infectadas pelo HIV, sem tratamento, evoluem para uma grave disfunção do sistema imunológico, à medida que vão sendo destruídos os linfócitos TCD4+, uma das principais células-alvo do vírus (BRASIL, 2019).

Desta forma, este agravo representa um problema de saúde pública de grande relevância em função do seu caráter pandêmico e de sua transcendência, pois desde seu primeiro caso descrito no Brasil em 1980 vivemos uma epidemia desta doença com 966.058 casos notificados em 2019, e nesse mesmo período foram notificados 332.505 casos entre mulheres de idade adulta e 633.462 casos em homens (BRASIL, 2019).

De 2007 até junho de 2019, foram notificados no Sinan 300.496 casos de infecção pelo HIV no Brasil, sendo na região Nordeste, corresponde a $8,9 \%$ de todos os casos de aids no Brasil, sendo Pernambuco o estado com maior incidência com 28.152 casos. Recife, a capital de Pernambuco, possui a maior concentração dos casos com cerca de $40,0 \%$ de todo o Estado, a região metropolitana com $30,8 \%$ e os interiores com 37,7\% (BRASIL, 2019).

A infecção pelo vírus da imunodeficiência humana (HIV) passa a ser considerada uma condição crônica de saúde e com potencial controle endêmico a partir da implementação da terapia antirretroviral de alta atividade (TARV). Esta trouxe um considerável avanço no tratamento da aids relativo às ações do vírus HIV no organismo. A reconstituição do sistema imunológico e a redução de doenças secundárias foram resultado desta terapia iniciada em 1996 no Brasil. Mesmo, diante da relevância dessa patologia houve um aumento de 15 anos da expectativa de uma melhoria da qualidade de vida relacionada a vida dos pacientes (CHRISTON HPP, 2010).

Esse tipo de terapia ocasiona diversos sintomas indesejados estão associados aos efeitos adversos da farmacoterapia do HIV. Portanto, o risco de contrair novas infecções é alto devido à fisiopatologia desta doença, podendo variar de acordo com a população, acarretando num impacto negativo nesta terapia especificamente (PAULA CC, et al., 2012).Sabe-se que a adesão ao TARV melhora os resultados clínicos, controla o avanço da doença e diminui a taxa de mortalidade, o que, supostamente, deveria resultar em uma melhoria da qualidade de vida dos pacientes (CHIOU PY, et al., 2006).

Em contrapartida a esses benefícios, os efeitos colaterais da TARV incluem fadiga, náuseas, vômitos, diarréia e lipodistrofia. Esses sintomas contribuem para a descontinuidade da medicação, que resulta no aumento da carga viral no sangue, diminuição da contagem dos linfócitos T CD4+ (CD4). Isso pode aumentar a resistência do HIV aos medicamentos, resultando em uma falha no tratamento, infecções oportunistas e desperdício de investimento. (REMOR E, et al., 2007; RUIZ-PÉREZ L, 2006). 
O reconhecimento da importância da terapia antirretroviral (TARV) para a prevenção da transmissão, diminuição da morbimortalidade e melhoria da qualidade de vida, traz aos serviços públicos de saúde desafios programáticos e organizacionais de várias ordens. O monitoramento das várias etapas do cuidado dessas pessoas e o desenvolvimento de estratégias de intervenção para garantir o acesso oportuno e a manutenção da adesão ao tratamento estão no centro desses desafios, sendo fundamentais para a melhoria da resposta brasileira à epidemia (MENDES EV, 2005).

A Principal forma de prevenção da aids é através do uso do preservativo. Neste sentido, as mulheres apresentam uma grande desvantagem na prevenção das doenças sexualmente transmissíveis e da aids, pois o uso do preservativo é algo de difícil negociação nas relações entre homens e mulheres, principalmente quando se trata de relações estreitas e duradouras (DOURADO I, et al., 2006).

Portanto, em uma relação estável entre um casal, exigir o uso de preservativo com outra finalidade que não a contracepção implica para a mulher em riscos que perpassam por questões ligadas à afetividade, como a quebra de confiança entre o casal, bem como, por questões culturais, como os rótulos atribuídos às mulheres que mostram conhecimento e iniciativa na esfera sexual e mesmo o risco de perder 0 apoio financeiro do parceiro, caso dependa dele (SANTOS MAPS, et al., 2005).

Assim, ao se discutir a adesão e a não adesão ao antirretroviral é necessário ressaltar que a aids é uma doença crônica, sem cura conhecida até os dias atuais, acarretando o uso contínuo de medicamentos como forma de prolongar a vida com mais qualidade. Neste sentido, o objetivo desse estudo foi verificar o perfil da mulher portadora de HIV/aids e sua adesão à terapêutica antirretroviral, no serviço de referência em um município do estado de Pernambuco.

\section{MÉTODOS}

Trata-se de estudo quantitativo, descritivo, retrospectivo e documental no qual a população do estudo foi constituída por mulheres portadoras de HIV/aids que iniciaram o tratamento no serviço de referência em um município do estado de Pernambuco, no período de Janeiro 2014 a Dezembro de 2017.

Por se tratar de pesquisa de dados em prontuários foram incluídas as fichas de atendimento de mulheres portadoras de HIV/aids com idade igual ou superior a 18 anos e que contraíram a doença por qualquer forma de transmissão: sanguínea, sexual ou vertical. Foram elencados como critérios de exclusão: as fichas de mulheres portadoras de HIV/aids onde a escrita estivesse ilegível ou incompleta por qualquer que seja o motivo, bem como, das que foram a óbito no período determinado do estudo.

O instrumento de coleta de dados compreendeu um formulário semiestruturado, elaborado pelos pesquisadores. As informações sobre as variáveis de interesse dos componentes do corte transversal foram obtidas por meio do instrumento de coleta citado, cujos dados foram organizados por blocagem (dados sociodemográficos, hábitos de vida, dados complementares do caso e dados da infecção pelo HIV/aids).

Inicialmente, foi criado um banco de dados próprio para a pesquisa, utilizando o programa Microsoft Excel, versão 2010, onde as informações foram armazenadas em processo de dupla entrada. Posteriormente, os dados passaram por uma checagem (validate) para detectar possíveis incongruências e/ou erro de digitação. A partir disto, os dados foram tabulados, processados e analisados e seus resultados apresentados sob a forma de tabelas.

Este estudo fez parte do projeto de pesquisa intitulado "Perfil da mulher portadora de HIV/aids e sua adesão ao tratamento" que foi apreciado e autorizado pelo serviço de referência onde o mesmo foi realizado e, concomitantemente, aprovado pelo Comitê de Ética e Pesquisa do Centro Universitário do Vale do Ipojuca sob o registro do parecer № 1.868.426. Os procedimentos utilizados na pesquisa obedeceram à resolução de número 466/12, que envolve pesquisas em seres humanos, respeitando-se o sigilo e a confiabilidade das informações, portanto, não contrariando nenhum princípio bioético.

\section{RESULTADOS}

Inicialmente havia uma lista com 2.100 pacientes infectados pelo HIV/aids. Desta foram selecionadas as mulheres, objeto do estudo, totalizando 211 portadoras desta patologia. Sendo que, 12 pacientes não foram 
incluídas no estudo por serem menores de 18 anos, restando 199 o número de participantes desta pesquisa. Destas, 14 não aderiram ao tratamento, 9 desistiram do tratamento e 4 foram diagnosticadas mais não quiseram aderir ao tratamento, segundo registro em prontuário.

Ao analisar a situação sociodemográfica destas mulheres observou-se que a maioria delas tem idades entre 30 - 39 anos $(29,1 \%)$, mais da metade estudaram até o ensino fundamental $(59,8 \%)$, se declararam pardas $(64,3 \%)$ e eram solteiras $(56,8 \%)$. Com relação à renda familiar das participantes, verificou-se um grande percentual de ignorado $(98,5 \%)$, levando-se a acreditar que o nível de preenchimento desta variável é muito ruim, prejudicando, portanto, a sua análise (Tabela 1).

Tabela 1 - Distribuição de mulheres portadoras de HIV/AIDS segundo variáveis sociodemográficas, $n=199$, 2014-2017.

\begin{tabular}{|c|c|c|}
\hline Variáveis Sociodemográficas & $\mathbf{N}$ & $\%$ \\
\hline \multicolumn{3}{|l|}{ Idade } \\
\hline $18-29$ & 54 & 27,1 \\
\hline $30-39$ & 58 & 29,2 \\
\hline $40-49$ & 47 & 23,6 \\
\hline $50-59$ & 31 & 15,6 \\
\hline 60 ou mais & 9 & 4,5 \\
\hline \multicolumn{3}{|l|}{ Escolaridade } \\
\hline Analfabeta & 22 & 11,1 \\
\hline Ensino Fundamental & 119 & 59,8 \\
\hline Ensino Médio & 50 & 25,1 \\
\hline Superior Incompleto & 0 & 0 \\
\hline Superior Completo & 1 & 0,5 \\
\hline Ignorado & 7 & 3,5 \\
\hline \multicolumn{3}{|l|}{ Raça/Cor } \\
\hline Branca & 58 & 29,2 \\
\hline Preta & 4 & 2 \\
\hline Amarela & 2 & 1 \\
\hline Parda & 128 & 64,3 \\
\hline Indígena & 2 & 1 \\
\hline Ignorado/Branco & 5 & 2,5 \\
\hline \multicolumn{3}{|l|}{ Renda Familiar } \\
\hline < de 1 salário mínimo & 2 & 1 \\
\hline De 1 a 3 salários mínimos & 0 & 0 \\
\hline De 4 a 5 salários mínimos & 1 & 0,5 \\
\hline > de 5 salários mínimos & 0 & 0 \\
\hline Ignorado & 196 & 98,5 \\
\hline \multicolumn{3}{|l|}{ Estado Civil } \\
\hline Solteira & 113 & 56,8 \\
\hline Casada & 31 & 15,6 \\
\hline Viúva & 18 & 9 \\
\hline União Estável & 31 & 15,6 \\
\hline Divorciada & 3 & 1,5 \\
\hline Ignorado & 3 & 1,5 \\
\hline
\end{tabular}

Fonte: Melo SSBM, et al., 2020.

Quanto ao estilo de vida destas mulheres, identifica-se que segundo dados dos prontuários, mais da metade relataram não fazerem uso de álcool $(53,8 \%)$, não serem fumantes $(58,8 \%)$ e não fazerem uso de drogas ilícitas $(64,8 \%)$. Apesar de ter sido verificado um percentual significativo de respostas ignorado/branco destas três variáveis $(25,1 \%),(28,1)$ e $(26,6 \%)$, respectivamente, esses valores não interferiram nos achados deste estudo. No entanto, chama a atenção, pela gravidade da doença, um valor significativo de mulheres que declararam fazerem uso de álcool $(21,1 \%)$, contribuindo ainda mais para a sobrecarga, principalmente, da atividade renal e hepática (Tabela 2). 
Tabela 2 - Distribuição de mulheres portadoras de HIV/AIDS segundo estilo de vida. $\mathrm{n}=199,2014-2017$

\begin{tabular}{lcc}
\hline Estilo da vida & $\mathbf{N}$ & $\%$ \\
\hline Consumo de bebidas alcoólicas & & 21,1 \\
\hline Sim & 42 & 53,8 \\
Não & 107 & 25,1 \\
lgnorado/ Branco & 50 & 13,1 \\
\hline Tabagista & 26 & 58,8 \\
\hline Sim & 117 & 28,1 \\
Não & 56 & 8,5 \\
Ignorado/ Branco & & 64,8 \\
\hline Uso de drogas ilícitas & 17 & 26,6
\end{tabular}

Fonte: Melo SSBM, et al., 2020.

$\mathrm{Na}$ avaliação da presença de comorbidades associadas, verificou-se o registro de doenças agudas e crônicas. As que prevaleceram foram a caquexia com (3,0\%), a tuberculose (1,5\%), as doenças sexualmente transmissíveis (1,5\%), as doenças mentais (1,0\%). Chama a atenção ao fato de que em 180 prontuários analisados não havia o registro de nenhuma doença associada, o que leva a acreditar que ou o nível de registro é ineficiente ou realmente não existia doenças associadas ao agravo em questão nestas pacientes, o que é questionável, baseados em relatos literários (Tabela 3).

Tabela 3 - Distribuição de mulheres portadoras de HIV/AIDS segundo comorbidades associadas. $n=199$, 2014-2017.

\begin{tabular}{lcc}
\hline Comorbidades associadas & $\mathbf{N}$ & $\%$ \\
\hline Diabetes & 1 & 0,5 \\
Doenças Mentais & 2 & 1,0 \\
IST's & 3 & 1,5 \\
Hanseníase & 0 & 0,0 \\
Tuberculose & 3 & 1,5 \\
Caquexia & 6 & 3,0 \\
Hepatite & 1 & 0,5 \\
Dermatite persistente & 0,5 \\
Síndrome de Stevens-Johnson & 1 & 0,5 \\
Herpes Zóster & 1 & 0,5 \\
Sem registro em prontuário & 1 & 90,5 \\
\hline Total & 180 & $\mathbf{1 0 0 , 0}$ \\
\hline
\end{tabular}

Fonte: Melo SSBM, et al., 2020.

Referente a forma como a doença foi adquirida, constatou-se que a principal forma de aquisição da infecção, de acordo com os prontuários, foi por relação sexual (69,8\%), seguidas da transmissão vertical $(1,0 \%)$ e por acidente com material biológico (0,5\%). Outro fato importante, que merece destaque, foi à constatação de que em 57 pacientes não havia o registro em prontuário da via de transmissão da doença, reforçando a tese de que o registro em prontuários ainda é ineficiente (Tabela 4).

Tabela 4 - Distribuição de mulheres portadoras de HIV/AIDS segundo forma de adquirir a doença. $n=199$, 2014-2017.

\begin{tabular}{lcc}
\hline Via de transmissão & $\mathbf{N}$ & $\%$ \\
\hline Transmissão Vertical & 2 & 1,0 \\
Transmissão Sexual & 139 & 69,8 \\
Acidente com material biológico & 1 & 0,5 \\
Sem registro em prontuário & 57 & 28,6 \\
\hline Total & $\mathbf{1 9 9}$ & $\mathbf{1 0 0 , 0}$ \\
\hline
\end{tabular}

Fonte: Melo SSBM, et al., 2020. 
Com relação à adesão terapêutica antirretroviral e ao abandono do tratamento nota-se que 2,0\% não aderiram contra $91,0 \%$ que aderiram ao uso destes medicamentos. Entretanto, das 181 mulheres que inicialmente aceitaram se tratar 9 abandonaram o tratamento, segundo relato em prontuário, totalizando um percentual de $4,5 \%$. Fica aqui, mais uma vez, registrada a constatação da pouca importância que ainda é dada pelos profissionais de saúde dos registros de todo procedimento/atendimento que seja feito aos pacientes acompanhados nos serviços de saúde, uma vez que nos deparamos com achados do tipo, 14 mulheres não continha em seus prontuários a informação sobre seu tratamento (Tabela 5).

Tabela 5 - Distribuição de mulheres portadoras de HIV/AIDS segundo adesão a terapêutica antirretroviral e abandono do tratamento. $n=199,2014-2017$.

\begin{tabular}{lcc}
\hline Adesão e abandono do tratamento & $\mathbf{N}$ & $\%$ \\
\hline Adesão ao tratamento & & 91,0 \\
Sim & 181 & 2,0 \\
Não & 4 & 7,0 \\
Sem registro em prontuário & 14 & \\
\hline Abandono do Tratamento & & 4,5 \\
Sim & 9 & 86,4 \\
Não & 172 & 9,1 \\
\hline
\end{tabular}

Fonte: Melo SSBM, et al., 2020.

\section{DISCUSSÃO}

Atualmente a epidemiologia da aids no Brasil caracteriza-se pela heterossexualização, feminilização, faixa etária jovem, baixo nível de escolaridade e pauperização da doença. Estes dados preocupam o sistema de saúde em vigilância epidemiológica, pois a um risco de aumentar o número de casos de infectados, por se tratar de uma população em atividade sexual e em idade fértil, que relacionado aos fatores ambientais e sociais, aumentam a vulnerabilidade à doença (ANDREOLLI A, 2008). Baseado no boletim epidemiológico 2019 do Ministério da Saúde, os achados sobre o perfil sociodemográfico das mulheres que participaram deste estudo seguem aos encontrados a nível nacional, ou seja, acompanham a evolução das características da população atingida pela doença no Brasil (BRASIL, 2016).

Em relação às características predominantes, as mulheres têm idade entre 30 e 39 anos, são pardas e com pouca escolaridade. A grande maioria ignorou a renda familiar no prontuário, ressaltando que $1,0 \%$ tinha registro de renda familiar inferior a um salário mínimo. Baseado no boletim epidemiológico 2018 do Ministério da Saúde, a faixa etária de 30 a 39 anos, que em 2007 era de dez casos em mulheres, passou para 25 casos em mulheres em 2017, com variação de 55\% (BRASIL, 2017). Demostrando que há crescente número de mulheres sendo contaminadas e com isso a alteração da epidemia ao longo dos anos (UNAIDS,2017).

O aumento da proporção de casos de HIV/aids em mulheres com menor escolaridade tem sido denominado pauperização, considerando-se nesse contexto a escolaridade como marcador da situação socioeconômica (FELIX G e CEOLIM MF, 2012). A população mais pobre sofre pela dificuldade de acesso a serviços básicos e/ou de qualidade, como educação e saúde, acarretando uma situação de vulnerabilidade social (FONTE VRF, et al., 2012).

Portanto, pode-se inferir, para este estudo, que todas as pessoas estavam vulneráveis a adquiri-la, independentemente da quantidade de anos estudados. No entanto, é uma informação que não se pode generalizar, visto que existe estudos que demostram o contrário, que o nível de escolaridade influência nos comportamentos de risco, dentre eles o sexo seguro (SILVA MM, et al., 2013).

Um pouco mais da metade das participantes se declaram pardas diferentes do encontrado por outros autores que relataram maior proporção de brancos entre doentes com HIV/aids na região nordeste do Brasil (BENSEN A, et al., 2011). Historicamente, indivíduos pardos e com baixa escolaridade possuem menos acesso aos serviços de saúde e à educação, o que, de modo direto, relaciona-se às informações sobre as formas de transmissão das doenças e meios de prevenção (GALVÃO JMV, et al., 2017). É possível que as diferenças acima descritas estejam relacionadas às diferentes localidades onde os estudos foram realizados. Além disso, em Pernambuco 38,4\% da população geral é constituída por pardos (BRASIL, 2016). 
Com relação ao estado civil das mulheres estudadas houve o predomínio de solteiras com $56,8 \%$ do total. Em relação às características predominantes no estudo, no que se refere ao uso de drogas, foi observado que quase $21,1 \%$ das participantes faziam uso de bebidas alcoólicas. Esse resultado também foi evidenciado em estudos de diversos autores, visto que a associação do uso de bebidas alcoólicas está interligada com HIV/aids (SEVERO NPF, et al., 2007).

Cerca de $13,1 \%$ das mulheres informaram ser fumantes contra $58,79 \%$ que negavam o tabagismo. Alguns estudos têm descrito a relação do tabagismo com o risco de desenvolver tuberculose, há evidências suficientes de que a TB, o HIV/aids e o tabagismo criam uma sinergia na carga de doença. Fumantes com TB e HIV/aids podem não ter acesso aos cuidados de saúde ou aos apoios sociais necessários para as mudanças de comportamento em saúde (NOVOTNY T, et al., 2017). Quase 8,5\% das pacientes referiam uso de drogas ilícitas. Este achado possivelmente está relacionado ao uso cada vez maior de drogas em nossa sociedade, constituindo-se em um problema importante de saúde pública.

No presente estudo a principal causa de aquisição do vírus HIV/aids foi à relação sexual sem proteção, dado semelhante a outras pesquisas na literatura (BRASIL, 2016). O alto percentual de casos adquiridos por relação sexual $69,8 \%$, somados aos $1,0 \%$ que foram adquiridos por meio da transmissão vertical e $0,5 \%$ por acidente de material biológico é preocupante, associado também ao aumento da possibilidade de transmissão dos vírus da hepatite $\mathrm{B}$ e $\mathrm{C}$. Uma de suas principais recomendações refere-se aos acidentes por material perfurocortante, que devem ser avaliados imediatamente após o ocorrido, utilizando-se de condutas, que diferem para cada tipo de vírus, para verificação das exposições ocupacionais a materiais biológicos potencialmente contaminados com o HIV, vírus da imunodeficiência humana; o HBV, vírus da hepatite B; e o HCV, vírus da hepatite C (BRASIL, 2010).

Ainda, em relação às características das doenças associadas ao HIV/aids em cerca de 1,5\% das mulheres havia o registro de doenças sexualmente transmissíveis, mostrando a necessidade de um incremento nas medidas de prevenção contra essa patologia neste grupo de estudo. Algumas IST são assintomáticas. Algumas IST são assintomáticas. Assim, os indivíduos procuram os serviços de saúde apenas na presença de sintomas, perpetuando a cadeia de disseminação, reforçando a importância do rastreamento para 0 controle dessas doenças e prevenção de infecção pelo HIV/aids (CODES JS et al., 2006).

Apesar do grande aumento do número de pessoas com acesso ao tratamento antirretroviral, a cobertura terapêutica ainda é inferior a 50\% em todo continente (MARIZ CDA, et al., 2014). O uso do tratamento antirretroviral está ligado diretamente à melhora imunológica, o que protege os indivíduos da aquisição de doenças oportunistas, entre elas a tuberculose.

Diante do exposto, vale salientar o registro de $1,5 \%$ de mulheres deste estudo que apresentou como comorbidade a tuberculose. Existem evidências suficientes sobre a associação entre tuberculose e HIV/aids uma vez que a imunodepressão, e consequente resposta imune celular com menor eficácia, predispõem os indivíduos à progressão da infecção latente pelo M. Tuberculosi (BOFFO MMS, et al., 2014).

Sendo assim, a coinfecção TB/HIV é reconhecidamente nociva, com alto risco de mortalidade nos pacientes acometidos, sendo fundamental sua prevenção, detecção precoce e tratamento. Porém os registros nos prontuários apontavam que uma proporção de 90,5\% ignora essa alternativa, em sua maioria, não estavam disponíveis nos prontuários médicos.

O alto percentual de variáveis sem registro sobre comorbidades é preocupante, principalmente, por se tratar de informações relativas às características de doenças associadas. Estas informações são ignoradas pelos profissionais de saúde levando-nos ao desconhecimento da real situação dessa população. Essa problemática tem sido fator limitante para outros estudos que também analisaram dados de prontuários (GUIMARÃES NB, et al., 2009).

Considerando que 2,0\% não aderiram ao uso de medicamentos antirretroviral uma vez que a adesão é um ponto crucial para o sucesso da terapia antirretroviral, isto traduz-se em um fato preocupante. A adesão é entendida como o estabelecimento de uma atividade conjunta na qual o paciente não é um mero seguidor da orientação médica, mas entende e concorda com a prescrição recomendada (MONTARROYOS UR, et al., 2014). Nessa perspectiva a adesão deve ser compreendida como um processo dinâmico multideterminado e de corresponsabilidade entre paciente e equipe de saúde. 
Isso torna necessária uma atenção especial na preparação das usuárias ao iniciarem o tratamento, a fim de que seus benefícios sejam compreendidos e que este assuma um significado positivo na vida dessas portadoras de HIV/aids. Mesmo tendo uma equipe multidisciplinar desenvolvendo uma estrutura de seguimento e de apoio psicossocial para a paciente ao longo do tratamento, se faz necessário esse reforço uma vez que os níveis de adesão tendem a diminuir após períodos prolongados de uso de medicamentos.

Com relação ao abandono de tratamento, têm-se o seguinte registro 4,5\% das pacientes abandonaram a terapêutica antirretroviral, 09 desistiram do tratamento e 04 foram diagnosticadas mais não quiseram aderir ao tratamento. Este fato merece toda a atenção uma vez que isto significa que as pacientes permanecem contaminantes por mais tempo e leva a um aumento da mortalidade. Tendo como possíveis causas o acesso aos serviços de saúde, contudo, os registros em prontuários não deixaram claras as verdadeiras causas do abandono.

Foram muitas as dificuldades encontradas no desenvolvimento desta pesquisa das quais destacamos a impossibilidade de obter todos os dados nos prontuários do local do estudo, em virtude do inadequado registro identificado, os quais seriam usados como denominadores para os cálculos da pesquisa, comprometendo de certa forma a análise de algumas variáveis do estudo.

Apesar das dificuldades encontradas, acredita-se que esse estudo trará contribuições importantes que devem alertar e colaborar para a melhoria da qualidade da atenção nos programas de HIV/aids do município em questão. Até o momento não existia em Caruaru pesquisa relacionada ao perfil da mulher portadora de HIV/aids e sua adesão ao tratamento, sendo este um estudo pioneiro. Estas contribuições devem beneficiar diretamente a equipe multidisciplinar, pois é sabido que controle de HIV/aids beneficia a todos.

\section{CONSIDERAÇÕES FINAIS}

O perfil dos participantes do estudo constituiu-se de mulheres adultas jovens, solteiras, pardas e com baixa escolaridade, em que a maioria não fazia uso de bebidas alcoólicas, drogas ilícitas e não eram fumantes. Essas apresentavam infecções sexualmente transmissíveis e tuberculose como umas das principais comorbidades, sendo a principal forma de aquisição a relação sexual. Identificou-se alto índice de aceitação do tratamento, porém, com registros significativos de abandono da terapêutica. Sendo assim, faz-se necessária a criação de um modelo assistencial voltado ao perfil das usuárias, principalmente àquelas que abandonaram o tratamento, com uma prática participativa, coletiva, com grupos educativos que promovam ações preventivas. Sugere-se o desenvolvimento de novos estudos que identifiquem e desenvolvam estratégias para superação de barreiras ligada à realidade de vida, individual e coletiva a fim de reduzir esses indicadores de abandono.

\section{REFERÊNCIAS}

1. ANDREOLLI A. As pessoas que vivem com HIV/AIDS: Uma revisão da literatura científica, RS. Monografia (especialização em saúde pública). Universidade Federal do Rio Grande do Sul, Porto Alegre, 2008; 06 p.

2. BRASIL. Secretaria de Saúde do Estado de Pernambuco. Secretaria Executiva de Vigilância em Saúde. Diretoria Geral de promoção, monitoramento e avaliação da situação de saúde. Perfil sociodemográfico e epidemiológico de Pernambuco. Pernambuco, dez.2010.

3. BRASIL. Ministério da saúde, boletim epidemiológico panorama epidemiológico da coinfecção TB-HIV no Brasil 2019. Secretaria de Vigilância em Saúde Ministério da Saúde - DCC Brasília, 2019; 50(26): 19-20.

4. BRASIL. Secretária de vigilância em Saúde, Ministério da saúde. Bol Epidemiol HIV/ aids. Brasília, DF. 2016.

5. BRASIL. Ministério da saúde, boletim epidemiológico HIV e Aids. Secretaria de Vigilância em Saúde Departamento de Doenças de Condições Crônicas e Infecções Sexualmente Transmissíveis - DCC Brasília: Ministério da Saúde, 2016.

6. BRASIL. Ministério da saúde, boletim epidemiológico HIV e Aids. Secretaria de Vigilância em Saúde Departamento de Doenças de Condições Crônicas e Infecções Sexualmente Transmissíveis - DCC Brasília: Ministério da Saúde, 2019.

7. BRASIL. Ministério da Saúde. Secretaria de Vigilância em Saúde. Coordenação-Geral de Desenvolvimento da Epidemiologia em Serviços. Guia de Vigilância em Saúde: volume único [recurso eletrônico] / Ministério da Saúde, Secretaria de Vigilância em Saúde, Coordenação-Geral de Desenvolvimento da Epidemiologia em Serviços. - 3ª́. ed. - Brasília: Ministério da Saúde, 2019.

8. BRASIL. Ministério da Saúde Brasil. Secretária- Executiva. Programa Nacional de DST e AIDS. Recomendações para terapia antirretroviral em adultos infectados pelo HIV. 7ª̣ edição. Brasília- DF: MS; 2017. 
9. BRASIL. Secretaria de Saúde do Estado de Pernambuco. Secretaria Executiva de Vigilância em Saúde. Diretoria Geral de promoção, monitoramento e avaliação da situação de saúde. Perfil sociodemográficos e epidemiológico de Pernambuco. Pernambuco, 2016.

10. BENSEN BA, et al. Clinical, radiological, and laboratory characteristics HIV/AIDS patients: Comparative study of HIVpositive and HIV-negativo impatient at a referral hospital. J Bras. Pneumol. 2011; 37(6):768-75.

11. BOFFO MMS, et al. Tuberculose associada a AIDS: Característica demográficas, clínicas e laboratoriais de pacientes atendidos em um serviço de referência do sul do Brasil. 2004; 30(2): 140-146.

12. CHIOU PY, et al. Programme of symptom management for improving quality of life and drug adherence in AIDS/HIV patients. J Adv Nurs. 2006; 20(2):169-9.

13. CODES JS, et al. Detecção de doenças sexualmente transmissíveis em ambientes clínicos e não clínicos na Cidade de Salvador, Bahia, Brasil. Cad Saude Publica 2006; 22(2): 325-334.

14. CHRISTON HPP. Alterações cognitivas na infecção pelo HIV e AIDS. Revista Associação Medicina Brasileira, 2010; 56(2): $242-7$.

15. DOURADO I, et al. Tendências da epidemia de AIDS no Brasil após a terapia antirretroviral. Rev Saúde Pública, 2006; 40: 9-17.

16. FELIX G, CEOLIM MF. O perfil da mulher portadora de HIV/AIDS e sua adesão à terapêutica antirretroviral. Rev Esc Enferm USP, 2012; 46(4):884-91.

17. FONTE VRF, et al. Conhecimento de gestantes de um hospital universitário relacionado à prevenção de DST/AIDS [STD/AIDS preventionrelated knowledge by pregnant women at a teaching hospital]. Revista de Enfermagem UERJ,2012; 20(4): 493-499.

18. GALVÃO JMV, et al. Demographic and socio-demographic profile of people living with HIV/AIDS. Rev Enferm UFPI, 2017; 6 (1): 4-8.

19. GUIMARÃES NB, et al. Estudo clínico-epidemiológico e laboratorial de pacientes co-infectados por tuberculose e AIDS. Rev. Para.med, Belém. Jul- Set 2009; 23(3):33-43.

20. MARIZ CDA, et al. Body mass índex in induviduals with HIV infection and factores associated with thinness and overweiglt/obesity. Cadernos de Saúde Pública, 2014; 27: 2009-2013.

21. MONTARROYOS UR, et al. Factores related to changes in CD4+ T-cell counts over time in patients living with HIV/AIDS: a multilevel analysis. PLos one, 2014; 9 (2): e84276.

22. MENDES EV. A construção social da atenção primária à saúde. Brasília (DF): Conselho Nacional de Secretários de Saúde - CONASS, 2015. 193p.

23. REMOR E, et al. Adaptação brasileira do "Cuestionario para la Evaluación de la Adhesión al Tratamiento Antiretroviral". Rev Saude Publica. 2007;41(5):685-94.

24. RUIZ-PÉREZ L, et al. Impact of demographic and psychosocial factors on adherence to antiretroviral treatment. Enferm Infecc Microbiol Clin. 2006;24(6):373-8.

25. NOVOTNY T, et al. HIV/ AIDS, tuberculose e tabagismo no Brasil: uma sindemia que exige intervenções integradas. Cad. Saúde Pública, 2017; 33 (3): 21-23

26. PAULA CC et al. Caracterização da morbimortalidade de crianças com HIV/AIDS em serviço de referência no Sul do Brasil. Revista Saúde (Santa Maria), 2012; 38 (2): 25-36.

27. SANTOS MAPS, et al. Risk factors for treatment delay in pulmonary tuberculosis in Recife, Brasil. BMC Public Health, 2005; 5 (25): 1-8.

28. SEVERO NPF, et al. Clinical and demographic characteristics of patients hospitalized with HIV in Brasil between 1994 and 2004. J. Bras. Pneumol., 2007; 33 (5): 565-571.

29. SILVA MM, et al. Caracterização epidemiológico dos casos de AIDS em pessoas com 60 anos ou mais, Pernambuco, Brasil, 1998 a 2008. Cad. Saúde pública. 2013; 29(10):2131-2135.

30. UNAIDS BRASIL. Programa conjuntos das Nações Unidas sobre HIV/ AIDS. 2017. 